\title{
Jourl'tial of Digital Imaiging
}

\section{Internet Consultations from a Remote Pacific Island: Impact of Digitized Radiologic Images on Referral Decisions}

\author{
Jinha M. Park, MD, PhD, ${ }^{1, *}$ Lynne Ruess, MD, ${ }^{1,4,5}$ Stephen C. O'Connor, MD, ${ }^{1,+}$ \\ Faheem Hussain, MD, ${ }^{1, \dagger}$ David Y. Oshiro, $\mathrm{BS}^{2}{ }^{2}$ and Donald A. Person, $\mathrm{MD}^{3,5}$
}

A study was carried out to determine whether digitized radiologic images added valuable information to Internet consultations from a remote Pacific Island. Chuuk State Hospital (Federated States of Micronesia) has limited film screen radiology, minimal ultrasound capability, and no radiologist. Providers initiate Web-based referrals for consultation or patient transfer. Digitized images (via low-cost digital camera or flatbed scanner) were uploaded to a Web site. Images were assessed for impact on referral decisions. A radiologist scored image quality and confidence (scale: 1-7). Of 97 referrals with images that were reviewed, 74 (76\%) image sets were abnormal, $20(20 \%)$ were normal, and $3(4 \%)$ were indeterminate. Median scores were 4 for image quality and 5 for diagnostic confidence. In most cases with abnormal radiology (52/74, 70\%), images were considered valuable. Radiologic images digitized with a low-cost camera or flatbed scanner provided valuable information for decision making in an Internet-based consultation and referral process from a remote, impoverished Pacific Island jurisdiction, despite relatively low image quality.

KEY WORDS: Telemedicine, teleradiology, internet communications, PACS, image capture

$\mathrm{O}$ UR INSTITUTION, the Tripler Army Medical Center, Honolulu, Hawaii, is the tertiary care facility charged with fulfilling a Congressional mandate to aid in the specialty health care needs of indigenous peoples of the United States Associated Pacific Islands (USAPI). Through the Pacific Island Health Care Project (PIHCP), the advanced medical needs of 1700 island patients have been addressed while providing graduate medical education opportunities for residency trainees. ${ }^{1}$ The project serves patients in the six jurisdictions of the USAPI (Formerly the US Trust Territories of the Pacific): American Samoa, the Federated States of Micronesia (FSM: Kosrae, Pohnpei, Chuuk, Yap), the Republic of the Marshall Islands, the Republic of Palau, Guam, and the Commonwealth of the Northern Marianas Islands. Chuuk State, FSM, with a population of approximately 53,000 , is one of the most impoverished in the Pacific Basin., ${ }^{2,3}$ Chuuk State Hospital is a 125-bed facility in Weno, the state capital, and it serves roughly 24 islands

${ }^{1}$ From the Department of Radiology, Tripler Army Medical Center, Honolulu, Hawaii.

${ }^{2}$ From the Information Management Division, Tripler Army Medical Center, Honolulu, Hawaii.

${ }^{3}$ From the Departments of Clinical Investigation and Pediatrics, Tripler Army Medical Center, Honolulu, Hawaii.

${ }^{4}$ From the Department of Radiology and Radiological Sciences, Uniformed Services University of the Health Sciences, Bethesda, Maryland.

${ }^{5}$ From the Department of Pediatrics, Uniformed Services University of the Health Sciences, Bethesda, Maryland.

* Present address: Department of Radiology, UCLA Medical Center, 10833 Le Conte Avenue, Box 951721, Los Angeles, California 90095-1721.

†Present address: Department of Radiology, Bldg 4-2817, Womack Army Med Center, Ft. Bragg, NC 28310, USA.

${ }^{\ddagger}$ Present address: Department of Radiology, Baystate Medical Center, 780 Chestnut St., Springfield, MA 01107, USA.

The views expressed herein are those of the authors and do not reflect the official policy of the Department of the Army, Department of Defense, or the US Government.

Correspondence to: Lynne Ruess, MD, Department of Radiology, Tripler Army Medical Center, 1 Jarrett White Road, Honolulu, Hawaii 96859; tel: 808-433-6669; fax: 808433-4688; e-mail: lynne.ruess@amedd.army.mil

Copyright (C) 2004 SCAR (Society for Computer Applications in Radiology)

Online publication 26 October 2004

doi: 10.1007/s10278-004-1022-6 
and 6 atolls. Basic hospital departments are in dire need of power, water, supplies, and medicines. There are no subspecialists. Most providers are medical officers who trained at the Pacific Basin Medical Officers Training Program in Pohnpei State, FSM, or the Fiji School of Medicine, or are expatriate physicians from the Philippines. Air travel is available one or two times per week.

Because of the shortage of hospital supplies and specialists, many patients are flown to neighboring islands like Guam, the Philippines, or Hawaii for complex surgical or medical conditions. Initially, consultations and referral decisions were made by airmail, letter, fax, and telephone. With the advent of e-mail, faster response times became possible. However, valuable data such as EKGs, patient photographs, and radiographic images was not easily accessible by specialists receiving the consultations. As a result, many patients were accepted for transfer, which often was found to be inappropriate. Patients with terminal illness or simple needs can be managed on the home island. The advent of the videophone added much needed clinical information with timely communication to the consults. ${ }^{4}$ However, the expense of such high-speed telecommunications and satellite systems quickly overwhelmed many of these island nations' health care budgets. The accessibility of Internet-enabled computers, affordable digital capture technology, and the availability of private Internet service via local telephone lines enabled the islands' medical officers to provide more useful information to specialists. Thus, in an effort to contain costs of this program, an Internet-based system was deployed in December 1997 to provide remote specialty consults for patient care from several Pacific Islands, including Chuuk State. ${ }^{1}$ Computer and Internet training of Chuuk healthcare providers was provided at a regional meeting where most providers first encountered this technology. Equipment was installed and additional training was provided by a site visit to Chuuk State Hospital. Since that time numerous providers have enthusiastically used this Web-based system with very few technical difficulties.

With improved remote consultation communication, the number of patients who must travel for care has decreased, with substantial cost savings to the island governments and to the PIHCP. ${ }^{1}$ The purpose of this study was to determine whether the addition of digitized radiologic images added valuable information to Internet consultations and referrals from Chuuk State, one of the remote Pacific Islands served by the PIHCP.

\section{MATERIALS AND METHODS}

The study was approved by the Institutional Review Board. Cases submitted using the secure Internet web site of the PIHCP by providers from Chuuk State Hospital between December 1997 and March 2002 were reviewed. A desktop Pentium II computer with $266 \mathrm{MHz}, 64 \mathrm{MB}$ RAM, 4.3 GB hard drive, $56 \mathrm{~kb}$ modem, $8 \mathrm{MB}$ video card, video capture board, and a 17 -inch 0.26 dpi color monitor was used for data entry. Image capture for photos, electrocardiograms, and radiographic images was done with either a Kodak DC 2651.6 megapixel digital camera (Rochester, $\mathrm{NY}$ ) or an Epson Expression 800 flatbed scanner with a transparency adapter (El Segundo, CA) at $72 \mathrm{dpi}$. All images were viewed as between 1 and 2 megapixels with 24-bit color range. These images were compressed in the Joint Photographic Experts Group (JPEG) compression format at the highest quality on Adobe Photoshop (San Jose, CA) before they were loaded onto the server.

Providers entered patient history, physical examination and laboratory results, and images on the Internet server. The program's Medical Director, an experienced clinician at our institution, reviewed each case. Cases were then forwarded to appropriate physician specialists and a radiologist if images were included and if radiologic consultation was deemed necessary. The specialists' comments were entered and the Medical Director evaluated each referral request to determine which patients would be transferred to our institution for further management. Guidelines for patient referral require that patients have a non-terminal, noncommunicable, correctable condition treatable at our institution.

For all cases with radiologic images, demographic data including patient age, gender, and whether the case was sent for consultation or it was a request for referral for treatment were recorded. The type and number of radiologic images was recorded along with the clinical question posed and the specialties to which the Medical Director forwarded the consult. A radiologist reviewed all the images retrospectively using a 17 -inch 0.26 -dpi viewing monitor set to True Color with a $1024 \times 768$ pixel area. The radiologist made a clinical impression of either normal, abnormal, or indeterminate and rated the confidence of the radiographic diagnosis on a scale of 1-7, with 7 being most highly confident. Each image was also rated for diagnostic quality, also on a 1-7 scale, with 7 being the highest quality. The diagnosis at the conclusion of the Internet consultation process was compared to the final diagnosis in those patients who eventually came to be evaluated at our insitution. The Medical Director reviewed 
the decisions retrospectively regarding approval for transfer and indicated whether the images were valuable in the decision-making process.

\section{RESULTS}

Of the 285 cases from Chuuk sent during the study period, 97 contained diagnostic imaging relevant to the consult. At time of presentation, the mean patient age was 31 years (range: 8 days old to 71 years old). There were 53/97 (55\%) males and 44/97 (45\%) females; of the 97 total cases, $34(35 \%)$ were children under 18 years of age. Cases were forwarded to the following medical and surgical specialties: surgery $41(42 \%)$, medicine $22(23 \%)$, and pediatrics 34 $(35 \%)$. Fourteen of $97(14 \%)$ cases included chest radiographs that were evaluated to ruleout pulmonary tuberculosis as a screening test prior to commercial airline travel when PPD testing was not available, not reliable, or when tuberculosis was suspected. Nearly all cases $89 /$ $97(92 \%)$ were referral requests for management not achievable at Chuuk State Hospital.

Of the 97 cases with relevant images, most cases had radiographs, 2 had ultrasound images, and one patient had CT images obtained in Guam included with the consult. There were 74 (76\%) abnormal image sets, $20(20 \%)$ cases with normal images, and $3(4 \%)$ that were indeterminate. The mean and median scores for diagnostic confidence were 5 (range: 1-7) and those for image quality were 4 (range: $2-7$ ). Fifty-five $(57 \%)$ image sets were deemed valuable to the decision-making process. Fifty two of $74(70 \%)$ abnormal image sets were valuable, but only 3 of $20(15 \%)$ normal image sets were valuable in the decision-making process.

Within the group of cases with valuable image sets, four cases involved consultation only, and the patients were not considered for referral. Twenty-four of $52(46 \%)$ cases with abnormal images that were considered for referral were denied on the basis of the Webbased diagnosis, and $24(46 \%)$ cases were accepted. Denials were commonly due to presumed tubercular disease, terminal disease, an uncorrectable condition, treatment not available at our institution, or poor surgical risk because of advanced disease. Twenty two of 74 (30\%) abnormal image sets did not influence decision making. Eight of these 22 (36\%) cases were denied.

Forty-nine of the total $97(51 \%)$ cases were accepted, $44(45 \%)$ were denied, and 4 were consultations only. Thirty-seven of $49(80 \%)$ patients arrived for treatment, $5(10 \%)$ patients died before traveling to our institution, 6 (12\%) were lost to follow-up, and 1 (2\%) declined referral. The final diagnosis was consistent with the accepting diagnosis in 34 of 37 patients $(92 \%)$. Thirty-three $(92 \%)$ were treated and returned home, and $3(8 \%)$ died during evaluation and treatment at our institution.

\section{DISCUSSION}

The Internet has become widely available around the world, even to the remotest islands in the Pacific Ocean. Medical care is severely limited in these remote areas, and access to specialties is scarce because of the expense and relative infrequency of available air travel. Through the implementation of an Internetbased consultation system, health care providers in a remote island hospital can consult with specialists at a tertiary care facility 3700 miles away.

All eleven sites in the PIHCP have a low-cost digital camera and a flatbed scanner available to digitize radiographs. At sites with ultrasound, practitioners photograph a still image from the monitor or scan printed images. We chose to evaluate the digitized images from Chuuk as a representative site of the program for several reasons: (1) it was the site of the first workstation; (2) its fragile infrastructure; (3) it is impoverished; and (4) it is a site of relatively frequent consults and referrals because of the relatively large population of the area. Yet even under these conditions, and with modest computer equipment, we found that the digitized images, particularly abnormal images, provided valuable information for decision making in our Internet consultation and referral process. The normal images were less often valuable to the consult decision-making process. They were frequently added after acceptance, as they were necessary to help exclude pulmonary tuberculosis and were required before patient travel was allowed on commercial airliners. 
Our interpreting radiologist has been practicing in a filmless environment and reviewing Internet consults for over 5 years, which may account for the relatively high confidence ratings despite low image quality. Confidence in reading such images is important when participating in such a program. We had similar results of high diagnostic confidence and low image quality when we had both experienced radiologists and radiology residents compare abnormal pediatric chest radiograph images digitized using the same two modalities available to the Chuuk State providers (digital camera and flatbed scanner with transparency adapter). ${ }^{5}$ In that study, we demonstrated that radiologists had higher diagnostic confidence when radiographs were digitized from the flatbed scanner rather than with the digital camera, and that diagnostic confidence remained high despite low image quality. As a result, we asked the referring providers at all of the Pacific Island sites to preferentially use the flatbed scanner for their consult images starting in late 2000.

Besides the obvious individual patient benefit in these cases, these Internet consultations have had great educational benefit for both the residents in our institution and the health care providers in Chuuk and the other Pacific Islands participating in the program. Many of these cases demonstrate the natural history of neglected or unusual tropical diseases, and they provide interesting and complex teaching cases. Providers themselves are brought up to date with the standard of care and are introduced to new procedures and therapies. Although there are a number of illustrative cases that could be cited to underscore the utility of the simple store-and-forward methodology described here, one is especially poignant. In December 1998, a 15-month-old girl was referred from Chuuk for evaluation of a large head with frontal bossing. She also had bowed legs, and the referring physicians felt that she had hydrocephalus as well as osteogenesis imperfecta. Initially only photographic images (Fig 1) of the patient were included with the consultation. The Medical Director requested radiographs of the skull and the lower limbs. The case was forwarded to the child neurologist and pediatric radiologist. When it was determined that the child's anterior fontanelle was patent, the radiologist asked lo-

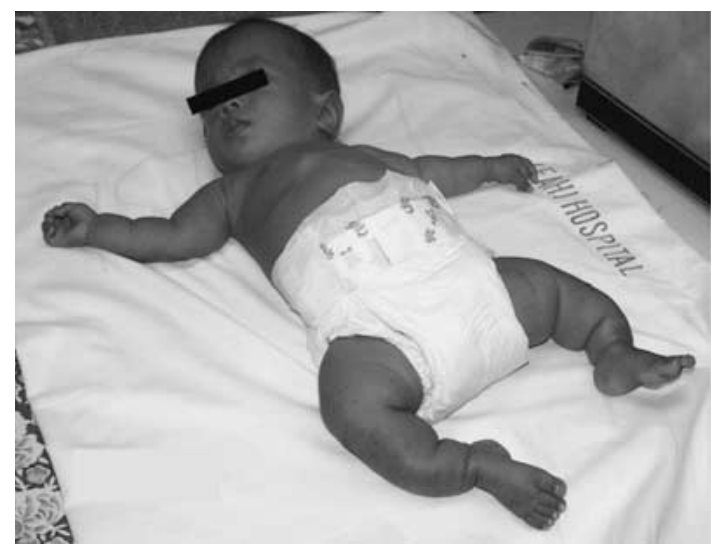

Fig 1. Photograph of a 15-month-old baby referred for evaluation of frontal bossing and possible osteogenesis imperfecta.

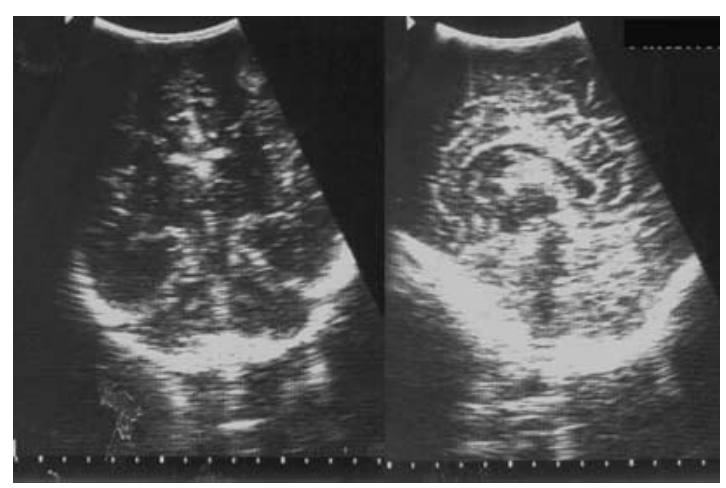

Fig 2. Ultrasound images of the brain via the anterior fontanelle show no hydrocephalus. Images were scanned from paper images obtained by Chuuk providers.

cal physicians about the availability of ultrasound. An obstetrical ultrasound unit had recently become available. Our radiologist posted a set of simple instructions to include probe placement and patient position, as well as sample ultrasound images demonstrating normal and abnormal ventricular size. Remarkably, Chuukese providers were able to obtain images which ruled out hydrocephalus (Fig 2). Unfortunately, the radiographs of the legs confirmed osteogenesis imperfecta (Fig 3), thus disqualifying the child from care as she had an uncorrectable condition. This case is especially instructive in that even in an impoverished setting, the Chuuk providers were anxious to expand their acumen and develop new diagnostic capabilities. This case has been used as the 


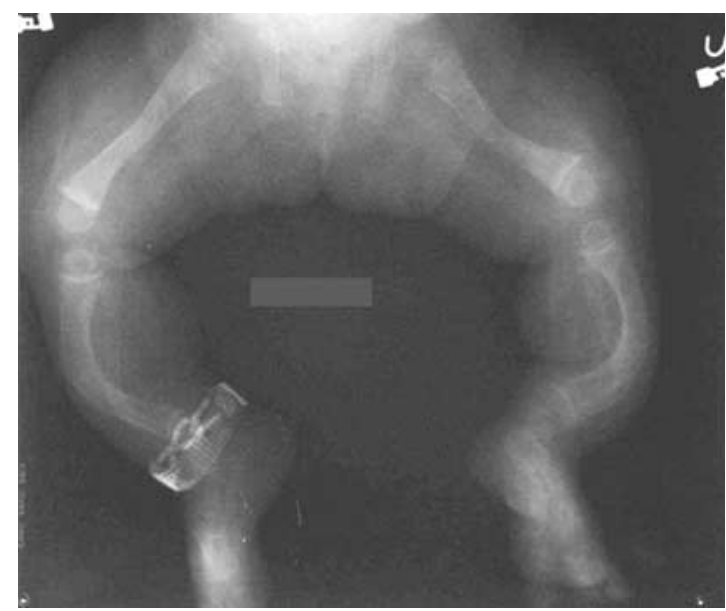

Fig 3. Digitized radiograph of the lower limbs shows osteopenia and multiple fractures consistent with osteogenesis imperfecta. Despite low image quality, the diagnosis can be made with confidence.

model for subsequent evaluation of infants with presumed hydrocephalus. In the past, such patients would have been transferred to our institution at great expense and without regard to our ability to deliver definitive care.

Limitations of our study include problems with a retrospective review. The radiologist and the Medical Director who made decisions about images for this study had seen many of the cases (radiologist) or all of them (Medical Director) when they were initially presented. In many cases, both knew the final diagnosis and the outcome. To minimize the bias that this knowledge could cause, the radiologist's diagnosis and the accepting diagnosis that were recorded on the original referral were used for all cases that the radiologist had seen previously. Selection bias limits comparison of the small group with both an accepting diagnosis and a final diagnosis, because the latter could only be determined for those patients selected for transfer to our institution for further evaluation and treatment.
With advances in technology, newer lowpriced digital cameras and flatbed scanners offer higher resolution than the equipment used in our study. Nevertheless, based on our experience and this study, we have no plans to change the hardware configurations at our current $\mathrm{Pa}$ cific Island locations. With high agreement between accepting diagnosis and final diagnosis, and a large number of radiographs deemed valuable in the consult process, we plan to continue using this low-cost system to add radiographic images to Internet consultations from remote Islands.

In conclusion, images digitized with a lowcost digital camera or flatbed scanner provided valuable information for decision making in an Internet consultation and referral process, despite low image quality. Application of this relatively inexpensive technology can be applied to health care enterprises providing consultations and referral decisions in a large network of remote health care units. This may lead to improved access to specialists in other remote parts of the world and continued unique educational experiences for physicians-intraining.

\section{REFERENCES}

1. Person DA: Pacific Island Health Care Project: early experiences with a Web-based consultation and referral network. Pacific Health Dialog 7:29-35, 2000

2. Government of the Federated States of Micronesia Web site. Available at: http://www.fsmgov.org/. Accessed December 26, 2002

3. Feasley VC, Lawrence RS eds: Pacific Partnerships for Health Washington DC, National Academy Press, 1998, pp 1-154

4. Yano V, Finan S, Dever G, et al: The PBMA and telemedicine in the Pacific: the first steps. Pac Health Dialog 4:81-84, 1997

5. Ruess L, Uyehara CFT, Shiels K, et al: Digitizing pediatric chest radiographs: comparison of low-cost off-theshelf technologies. Pediatr Radiol 31:841-847, 2001 